\title{
On the Development of Electronic Design Tools and Associated Guidelines for Supporting the Early Stages of the Design Process
}

\author{
Derya Ozcelik \\ Eindhoven University \\ of Technology \\ Postbus 513, $5600 \mathrm{MB}$ \\ Eindhoven, The \\ Netherlands \\ +31402475228 \\ d.ozcelik@tue.nl
}

\author{
Javier Quevedo- \\ Fernandez \\ Eindhoven University \\ of Technology \\ Postbus 513, $5600 \mathrm{MB}$ \\ Eindhoven, The \\ Netherlands \\ +31402472514 \\ j.quevedo.fernandez@ \\ tue.nl
}

\author{
Jos Thalen \\ University Twente \\ Postbus 217, 7500 AE, \\ Enschede, the \\ Netherlands \\ +31534893192 \\ j.p.thalen@utwente.nl \\ Jacques Terken \\ Eindhoven University \\ of Technology \\ Postbus 513, $5600 \mathrm{MB}$ \\ Eindhoven, The \\ Netherlands \\ +31402475254 \\ j.m.b.terken@tue.nl
}

\begin{abstract}
According to the user-centred design (UCD) philosophy, users should be involved as much as possible in the different stages of the design process, to optimize the likelihood that design proposals will be appreciated by users. In this paper, first we consider some challenges and questions that are raised by this general guideline. Next, we summarize outcomes of an ongoing project that addresses the question of when and how to involve the user in the design process. We report UCD opinions and practices in industry. In addition, we present work in progress aiming to develop tools and guidelines that should support design departments in industry to develop design proposals that optimize the fit-to-context. This includes a study investigating the effects of realism and animation of concept representations on user feedback. These studies help us to get a better understanding of when and how to involve users in the design process.
\end{abstract}

\section{Keywords}

Design process; User-centred design; Concept generation; Concept development; Design tools and guidelines; Prototyping; Sketching; VR

\section{INTRODUCTION}

According to user-centred design philosophy 32 and related views such as people-centred design 33 and

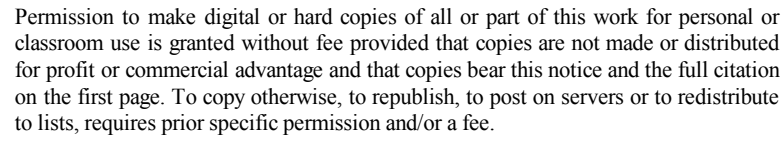

Permission to make digital or hard copies of all or part of this work for personal or classroom use is granted without fee provided that copies are not made or distributed for profit or commercial advantage and that copies bear this notice and the full citation on the first page. To copy otherwise, to republish, to post on servers or to redistribute to lists, requires prior specific permission and/or a fee.

DESIRE'11, October 19-21, 2011, Eindhoven, the Netherlands Copyright (c) 2010 ACM ISBN 978-1-4503-0754-3... \$10.00 human-centred design 918 , users should be involved in all stages of the design process, in order to give feedback on and guidance for design decisions, increasing the likelihood that the outcomes of the design process fit people's expectations and thus have less chance to fail in the market 27]. This includes the early stages of the design process, where the designer would like to find out whether a certain idea will be valuable to people. The main consideration to involve users in this stage of the design process is that users will be better able to judge whether something will be valuable to them than the designer. After all, it should fit to their context, and they are the experts on their context.

The user involvement in the early stages of the design process creates a problem for designers. On the one hand, the rough and sketchy representations, typical of the early stages of the design process, may fail to communicate the idea to users adequately. On the other hand, more sophisticated representations, that might communicate the early concepts more adequately, force designers to make decisions and fill in details that they would like to leave open at this early stage. In addition, such representations may elicit the wrong kind of feedback.

Traditionally, many designers therefore avoid including users in the very early stages of the design process. They believe that early representations of ideas and concepts are not suited to be communicated to users. In addition, if the question is whether a given concept will create value for users, many designers believe that users are not in a better position than they are themselves to form an opinion on this. They argue that users would have to project the idea towards a future context, and thus need to make a prediction that the proposal will fit to this future context, 
and that designers, having knowledge about how trends and user preferences evolve, in fact might be equally well or even better equipped to make such predictions.

These considerations challenge us to show that it makes sense to involve the users in the early stages of the design process and to develop tools, methods and guidelines for these early stages. We took up this challenge in the REPAR project, which stands for Resolving the Paradox in User-centred Design Process through Flexible Prototyping. In the current paper, we report on the first results of the REPAR project. We show that companies on the one hand see the need of involving users in the early stages of the design process but on the other hand they find it difficult to implement a good approach. Next, we describe work in progress on the development of tools and guidelines intended to support companies in implementing such an approach. The guidelines should answer the question of when and how to involve users in the early stages.

In order to get a good understanding of the challenges that we face and to put the questions in context, we make a distinction between two types of innovation, viz. radical and incremental innovation 31. In the case of radical innovation, designers come up with radically new products, for which there exist no precursors in the market. In the case of incremental innovation, typically there are already precursors in the market. Either a company may come up with a new version or release of a product or service it is already selling. Or it may want to enter an existing market.

The important difference between radical and incremental innovation is that in the latter case users already have experience with this type of functionality and therefore can inform the design team (usually mediated through the marketing department) about the current fit-to-context. The design efforts in such cases are usually devoted to adding new features and fixing usability flaws. Also, changes in styling (look-and-feel) may be made in this stage. On the other hand, in the case of radical innovation, there are no prior examples in the market, and it is precisely in this situation that, if we want to involve users in the early stages of the design process, we expect them to be able to make a projection towards the future, that is, to make a prediction that a certain design proposal would fit their future context. Obviously, even in the case of radical innovation, there are stages in the design process where the proposal starts to take concrete shape, and we can discuss the design proposal with users and investigate whether they consider it useful, easy-to-use and aesthetically pleasing. But it is precisely the early stages of the design process, before the ideas have started to take concrete shape, where we would like to get information about the fit-to-context of the design proposals.
In his book Design-driven Innovation, Verganti argues that successful design firms do not care about involving users in the early stages of the design process. Instead, they collect information about the user context in an indirect way, by involving user experts from diverse backgrounds, such as psychologists, psychiatrists, designers, architects, along with engineers and scientists who contribute expertise about new technologies. This information consists of insights about how people give meaning to their life through their everyday activities, and the purpose of the design process is then to come up with new visions on how people might give meaning, where this vision then is embodied in the artefacts that constitute the outcome of the design process.

In sum, while the Design-driven Innovation philosophy and the User-centred Design philosophy agree about the need to ground new design proposals in a good understanding of the future context of use, the difference between the two views is that according to User-centred Design philosophy users may contribute valuable information in the early stages of the design process. The challenge is mainly in how to help the designers and users to uncover the relevant information and bring it to the surface.

Tools and methods for user involvement in the early phases of the design process have emerged within the framework of User-centred Design and related approaches. Although these tools and methods are not only embraced but also further developed by big and well-known design consultancies and in-house design teams such as IDEO, Microsoft and BMW [33, an inspection of the design practice indicates that the involvement of users in the design process has not yet been well applied.

In the remainder of the paper, we present work in progress on the development of tools and guidelines for involving users in the early stages of the design process. First, we summarize a study of the current user-centred design practice in industry, to identify main bottlenecks for involving the user in the early stages of the design process. Next we present work on the development of tools and guidelines that should enable designers to create concept representations for co-reflection sessions with users in the early stages of the design process. This work includes a study that investigates which properties such early concept representations should have. We conclude by sketching directions for further work.

\section{PART I: EXPERIENCES FROM PRACTICE}

In order to understand the current state of user involvement within industrial practice we conducted a field study in collaboration with the research and development (R\&D) departments of four industrial companies. The companies, located in The Netherlands are active in different domains. The companies differ from each other in terms of the products they develop, their 
target market (consumer versus professional products) and their innovation style (radical innovations versus incremental innovations). A team of three researchers visited each company for a period of two weeks. The team not only observed the daily working routines of the R\&D teams but also conducted between 10 and 15 semistructured, 1 hour interviews with the team members, including designers, engineers and marketing people. Each visit was concluded with a workshop where the findings presented to the participants and discussed further.

Literature on user involvement in the design process suggests that end users are involved in the process in three ways: through what they say, through what they do and through what they make 25]. The say category involves methods like surveys and interviews, and is mainly managed by marketing departments, where a large amount of user data is gathered. The results are regarded useful as they indicate certain trends within the user population. The do category concerns monitoring users in product related contexts. These techniques reveal information about the behaviour of users in their use context. The make category refers to the cases where users are not only involved in identifying the problems but also creating the solutions. These sessions are done with a limited number of users in comparison to the surveys and interviews. The aim is to obtain in-depth information, thus details and divergences are more important than generalizations.

If we look at how the companies involve users early in the design process, we see that they rely most on tools and methods relating to what people say and what people do (see Table 1. The consumer needs are identified mainly according to what people say, although companies A and $\mathrm{B}$ also apply methods in the do category. In the concept generation phase companies $\mathrm{A}$ and $\mathrm{B}$ obtain information both through what people say, do and make; companies C and $\mathrm{D}$ do not involve users at all in the concept generation phase. It should be noted that the table does not express the iterative nature of the design process, which most companies apply. The concept generation involves several iterations resulting in prototypes with increasing realism, and companies A and B involve users in this stage through several methods in the say and do category. Concept evaluation involving potential end users through methods in the do category is then applied after the concept has been more or less consolidated.

In the interviews, most members of the R\&D teams stated to believe that users need to be involved in the early stages of the design process. However within their current practice early user involvement is not really applied due to certain challenges, relating both to the attitude of the company and external factors.

First of all, there is a difference in attitude towards early user involvement in the design process between those companies developing professional products and those developing consumer products. It seems more challenging for the companies in the field of professional products to achieve early user involvement, for several reasons. As these companies have been working in the same domain for a long time, they strongly believe that they know their users quite well through experience. Up to a certain extent their market knowledge is indeed quite extensive; however this attitude also brings the risk of ignoring changes in the market. Companies producing consumer products are more open to user involvement as their market changes quite fast. Furthermore, it is harder for the companies who develop professional products to reach end users. In the contacts with the customers, they have close contact with management making the purchase decisions, but not with the employees who are the real end users of the products. Finally, companies sometimes find it risky to involve clients early in the process, especially with products that are not entirely finished yet. If they show an early prototype, the client might not understand that this is just an early, unfinished prototype. Companies are afraid that early evaluations will affect the purchase decisions if the client gets a negative attitude towards the product due to the inferiorities of the prototype.

In the second place, some companies have a preference for involving user representatives instead of end users in the early stages of the design process. User representatives are

Table 1: Distribution of the tools and methods in different categories among the companies

\begin{tabular}{|c|c|c|c|c|c|c|c|c|c|c|c|c|c|c|c|}
\hline Phase & Say & $\mathrm{A}$ & $B$ & C & $\mathrm{D}$ & Do & $\mathrm{A}$ & $B$ & C & $\mathrm{D}$ & Make & $\mathrm{A}$ & $\mathrm{B}$ & $\mathrm{C}$ & $\mathrm{D}$ \\
\hline \multirow{3}{*}{$\begin{array}{l}\text { Identification of Consumer } \\
\text { needs }\end{array}$} & Interviews & $\mathrm{x}$ & $\mathrm{x}$ & $\mathrm{x}$ & $\mathrm{x}$ & Contextual Inquiry & & $\mathrm{x}$ & & & & & & & \\
\hline & Surveys & $\mathrm{x}$ & $\mathrm{x}$ & $\mathrm{x}$ & $\mathrm{x}$ & Shadowing & $\mathrm{x}$ & & & & & & & & \\
\hline & & & & & & Probes & $\mathrm{x}$ & $\mathrm{x}$ & & & & & & & \\
\hline \multirow{3}{*}{ Concept Generation } & Scenarios & $\mathrm{x}$ & & & & Contextual Inquiry & & $\mathrm{x}$ & & & Generative sessions & $\mathrm{x}$ & $\mathrm{x}$ & & \\
\hline & Focus Groups & $\mathrm{x}$ & $\mathrm{x}$ & & & Probes & $\mathrm{x}$ & & & & & & & & \\
\hline & & & & & & Shadowing & $\mathrm{x}$ & & & & & & & & \\
\hline \multirow[t]{3}{*}{ Concept Testing } & & & & & & Walkthrough & & $\mathrm{x}$ & $\mathrm{x}$ & & & & & & \\
\hline & & & & & & Heuristic Evaluation & & $\mathrm{x}$ & $\mathrm{x}$ & & & & & & \\
\hline & & & & & & User testing & $\mathrm{x}$ & $\mathrm{x}$ & $\mathrm{x}$ & $\mathrm{x}$ & & & & & \\
\hline
\end{tabular}


employees of the company who have good knowledge about the users and have the skills needed to use the product. Involving user representatives is preferred because of confidentiality, as it takes away the need to disclose ideas to the outside world. Also, it eases the communication, since user representatives are easier to reach, they can understand the language of designers better and they can express themselves better as they are familiar with design terminology. However, relying only on user representatives is risky because their needs, values and expectations are influenced by their past experiences as a member of the company. They provide valuable feedback, but they might not cover the complete spectrum of issues that end users would. Companies which are more relaxed in this respect, have a higher tendency to involve real end users.

In the third place, companies do not always possess the necessary knowledge about tools and methods that can be utilized for early user involvement. Companies pointed out that in some cases user involvement is postponed to later phases of the projects because they do not have tools to create easy and inexpensive prototypes that may communicate the experience or the envisaged functionality of a product to the end user in the early stages of the design process. As a result, some companies tend to postpone user involvement until the stage of usability evaluation, when more concrete proposals are available, which are suited to give users the opportunity to experience the concept. Yet, companies acknowledge that low fidelity and high fidelity artefacts (e.g. sketches, mock-ups, prototypes) provide different types of feedback. It is believed that high-fidelity representations impose a threshold for feedback, as users tend to be quite hesitant to give negative feedback. On the other hand, feedback gathered from low-fidelity prototypes might not be to the point as the user might not adequately understand the concept. Thus, designers find it difficult to decide which type of representation to use at what stage and what kind of feedback to collect.

The state of the art of UCD in practice indicates that although R\&D teams appear to believe in the benefits of involving users in the early stages of the design process, it is still very challenging to achieve. To overcome these challenges, they need better tools and guidelines. First of all, they are in need of tools that give them the ability to quickly and inexpensively create early concept representations which inform the end users about envisaged functionality and experience in the early phases of the design process. Secondly, they need guidelines, frameworks and methodologies to decide when, how and why these tools would bring added value.

In the following sections, in part II we present our work on the requirements gathering for and development of tools for the early stages of the design process; then, in part III, we present the first results of investigations of how design teams perceive the added value of these tools, and of how they should be applied to elicit certain types of feedback on early design representations.

\section{PART II: TOOL DEVELOPMENT: APPROACH}

The analysis of the workflow in the design process indicated that the creation of new concepts typically shows a pattern where designers first generate an idea and then start to translate it into a concept through a number of stages in which they elaborate the concept itself and explore its fit-to-context. In the REPAR project, it was postulated that the design process could be facilitated by a tool chain consisting of an electronic tool to support the initial stage where initial ideas are developed and explored through sketching and a tool for the later stages where the concept is further elaborated and the fit-to-context is explored through more hi-fidelity representations. In this section we present the approach to the development of these two tools.

At the beginning of the project, the situation for the envisaged tools for creating low-fi and hi-fi concept representations was different. For the tool for creating lowfi prototypes there was an already existing version available and the main target was to find out how to develop this further so that it would suit the needs of designers in industry. For the tool for creating hi-fi prototypes no existing version was available yet; instead, there was a set of VR technologies available for creating VR applications. As a consequence, the approach taken for the development of the two tools was also different, as will be described in the next subsections.

\section{Ila: Tools for creating low-fi prototypes}

Within the REPAR project, an electronic tool called Sketchify 21 is under development, to assist designers in quickly creating large amounts of expressive artefacts (Buxton's definition of sketch [5]), to be able to rapidly bring elements of the experience and functionality to the conversations with the end users. Sketching is considered one of the main activities in the early cycles of the concept generation stage [23], widely practiced in many design disciplines such as industrial design and architecture. In the early cycles of the concept generation stage, propositions are often represented in the form of sketches and low fidelity prototypes, because they are inexpensive, and if made carefully, can effectively communicate the envisaged concept. Sketching also assists creative processes by helping practitioners think and create new combinations of ideas 10 .

Various electronic sketching systems have been developed in recent years, such as SketchIT [28] used for the rapid creation of curves and surfaces, or SketchCAD [14 for creating technical drawings. There are also systems to sketch user interfaces like SILK 17, which enables the user to draw graphical user interfaces, recognizing the 
hand drawings, and automatically creating the appropriate widgets; or DEMIN [19], which focuses on sketching Web interfaces. DEMAIS [2] is a multimedia sketch-based editor that allows the user to define pages and navigation units, with the possibility of including rich media such as video or animations, similar to Microsoft's Sketchflow. Additionally, there are other tools designed to sketch interactive systems. Buck 22 supports prototyping of mobile devices, while D.tools 13 is used to design physical interaction prototypes. We also find integrated environments that can be used to sketch and develop complex interactive systems. Switcharoo 11 aims at supporting the development of physical interactive products. Calder and Phidgets support rapid prototyping of physical interfaces, and Max/MSP is one of the widely extended solutions that provide graphical development environments for music and multimedia. General-purpose programming languages such as Flash or Processing also provide environments for sketching and fast prototyping thanks to the large amount of libraries bundled, and the easiness and speed of programming.

In general, these solutions provide a large set of options to develop sketches and fast prototypes in the early stages of the design process. Despite this, we have identified certain limitations in them. As product design has grown in complexity, in order to materialize ideas, especially to communicate them to end users, designers need to build complex artefacts that enable the users to obtain a realistic experience of the functionality and the interface. For this purpose, designers often need to combine functionality from different design tools. Designers have very diverse backgrounds, depending on their field and expertise, and they often have trouble in moving from one tool to another. As we saw in the aforementioned systems, design tools are normally very specialized for a particular purpose. Thus, designers frequently have to rebuild artefacts from scratch by different software tools, to describe different aspects or characteristics of their concepts. None of the previous tools allow designers to easily and seamlessly combine tools from different design paradigms, such as painting, visual programming or hardware-software connectivity. As a result, none of them offer an integrated environment that supports the process from sketching the user interface, to testing it with real hardware. Additionally, extending the functionality of the tools towards using them in different domains is difficult and time consuming, and in some cases not even possible. Furthermore, the technology used by product designers changes rapidly 20], and new ones are constantly being developed and become easily accessible as their price drops quickly. Because of this, the user interfaces change significantly in a short period of time, and design tools can quickly become obsolete.
Sketchify aims at supporting the development of representations that give an impression of the overall user experience and the dynamics of the interaction. It attempts to achieve this by simplifying the interoperability and functionality of certain design tools, creating a platform that allows designers to easily mix and combine different design paradigms and different kinds of functionality. Sketchify supports simple exploration of different technologies, their possibilities and limitations, by simplifying their connectivity, inputs and outputs, making it easy for designers to play with and understand their behaviour and response. By supporting a wide variety and diverse set of design tools, Sketchify allows its users to choose those elements that fit better their skills, possibilities and mindset, giving them freedom to decide how they want to achieve their particular goal or solution, without being enforced to do it in a specific way (support for many paths, and many styles). Thanks to this coordination and ability to combine different design tools, Sketchify aims at stimulating their creativity through the synergetic use of the tools. Sketchify does not recreate a complete new sketching environment, but instead it reuses existing open source tools, such as visual editors and spreadsheets. Sketchify is easy to extend, since it is open source, and it allows designers to easily develop modules and services to connect new or existing tools and services into the platform.

Sketchify has been successfully used in educational settings 21. For the REPAR project, a new release was to be implemented with the purpose of being used by design practitioners in industry. Because the main aim of the new version was to adapt it to this new use context, we conducted a study in collaboration with the industrial partners of the project. The study was carried out using a user-centred design approach, in order to understand the real needs of our prospective users and the new context in which it would be used. 


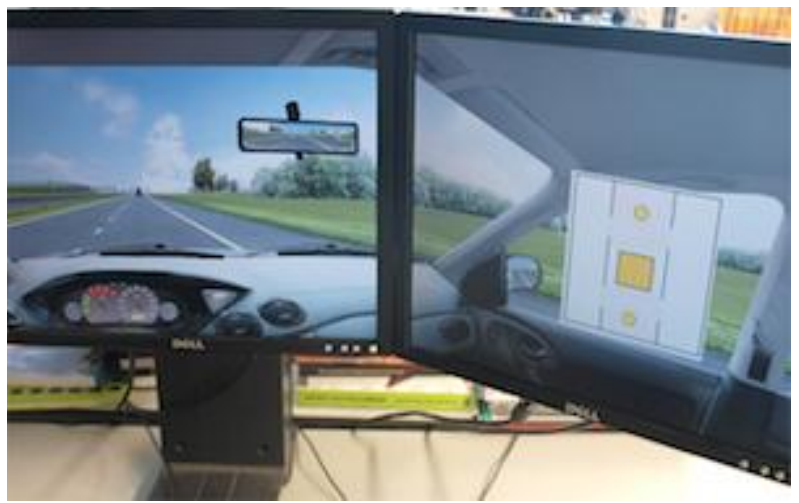

Figure 2: Sketchify connected to a driving simulator

\section{Approach}

\section{Collecting Preliminary Requirements}

During the field study in collaboration with our industrial partners (see Part I) the researchers interviewed a total of forty-nine designers, engineers and management personnel from different fields and backgrounds. In the interviews participants were asked about their design process and their design tools and methods. In relation to the sketching tool, the output of the field study was a list of preliminary requirements to address or improve some of the issues that had been shared by the practitioners, such as hardwaresoftware connectivity, visual end user programming or about sharing and collaboration.

\section{Sketchify as a Probe: Learning about the context of use and managing expectations}

The interviews were followed up with a workshop where designers were invited to see and experience a set of example artefacts and demonstrative projects (see Figures 1 and 2), in order to give them a better understanding of what the tool could be used for and to inspire them to provide early stage feedback about their vision and expectations. In order to make the demonstrative artefacts more meaningful and more representative of the

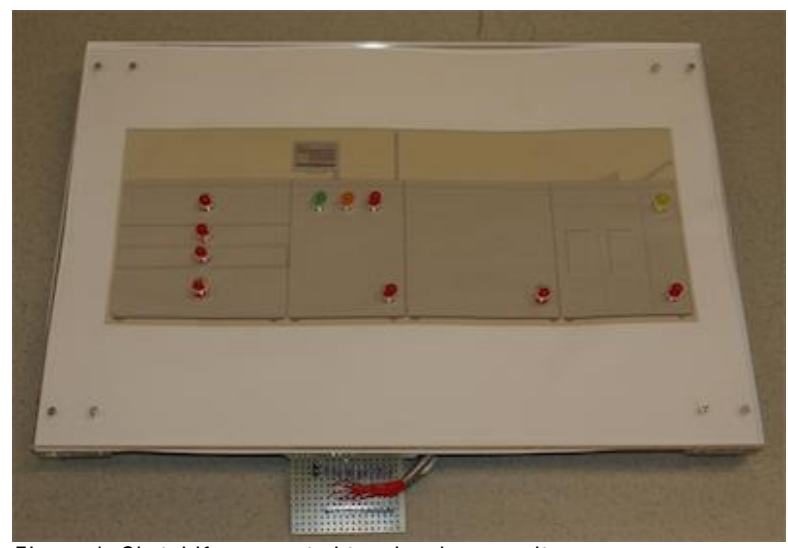

Figure 1: Sketchify connected to a hardware unit companies' needs, they were related to use cases provided by the industrial partners. During the workshop, the examples were presented one by one and discussed. Each demonstration artefact made use of different functional aspects of the sketching tool, and a follow up discussion was carried out after each demonstration and a final one in the end.

In order to gain a deeper understanding of the practitioners' needs, to collect more concrete data and evidence of which tool functionality was more relevant to each group of practitioners, a survey was completed by eighteen designers from different fields (including interaction designers, visual designers, product designers, experience designers, GUI designers, etc). The questions were grouped in three different categories: primary and secondary design tools (types of tools and frequently used functionalities and missed functionalities), context of use of the tools (phase of the design process, the audience) and tool complexity. The workshop insights and survey provided enough data to determine the characteristics of the tools.

\section{Iterative development approach}

The development was further informed by feedback from a few practitioners in the companies who were using the initial version of the tool and provided feedback about bugs and problems that they encountered. As the feedback was provided, the development team implemented the appropriate changes and returned to the practitioners with an updated version of the tool, turning the process into a cycle of development-feedback collection-development.

\section{Actual usage, in-the-wild evaluation}

A new version was released, which was made available to the companies for use in their daily practice. The release of the new version was supported by training sessions in which practitioners were given a brief tutorial and a set of practical exercises. Since the aim of this version was to fit to the real design context, in-the-wild evaluation was preferred over evaluation in a controlled setting. The evaluation is ongoing, and involves interested designers to use the tool in their regular work and to provide any kind of feedback that they consider useful. They will be consulted over a prolonged period of time about how the tool really fits their work.

\section{Ilb: Tools for creating hi-fi prototypes}

At a more advanced stage of concept development, capturing the functionality and the experience of a new product concept is likely to exceed the capabilities of lowfidelity prototypes, as low-fi representations leave too much room for imagination. For instance, it is difficult to let an end user experience the behaviour of a new car through a sketch or a static mock-up. The experience of the new product is affected by interactions with the use context (e.g. other users, other products, events, etc.), which should therefore be part of the prototype. 
We propose to use VR (Virtual Reality) technologies to create high-fidelity prototypes at a more advanced stage of concept development. VR technologies create an alternative reality in which worlds, objects and characters can be experienced that may not yet be available in reality. As such it allows end users not only to see the future product (which could also be achieved with a concept sketch or mock-up), but also to experience the product and the interactions with its use context. As such, 'highfidelity' refers to the integrality of these prototypes rather than for instance visual realism.

\section{Problem Definition}

Current applications of VR in product design exploit the ability of VR to allow non-existing products or environments to be experienced in a natural and realistic way. This is beneficial when the real world situation is too dangerous (e.g. a driving simulator, as described by 29]), when an environment needs to be controlled (e.g. in simulation and evaluation as described by 16]) or when physical prototyping is too expensive or simply not possible yet (e.g. virtual prototyping, as described by 3 ]). The examples of advantageous applications of VR technologies in the product development process illustrate the substantial set of applications already available for this field.

However, VR has yet to become a common tool amongst product designers in the early stages of design. We argue that the limited adoption of VR as a design tool is caused by the mismatch between VR design tools and VR design tool users (e.g. product designers). The majority of existing VR design tools originate from research in computer science, embodied in toolkits that extend programming languages with VR specific functions (see 34 for an extensive survey). Examples include VR Juggler 44, OpenTracker 24 and ARToolkit [15]. While these design tools provide excellent development platforms for experts and researchers, they are by no means usable for untrained product designers. Especially in the early stages of a design process, product designers can not be expected to invest time and effort in learning how to develop software or how to create complex 3D environments.

\section{Approach}

In this part of the REPAR project we aim to identify requirements for the development of usable and usefu/VR design tools. A user-centred approach is applied, in which product designers are considered the end users of the VR design tools and therefore form the main source of requirements. It should be noted that the focus of this research is not on developing the actual tools, but rather on using existing tools and toolkits as probes to elicit generic VR design tool requirements.

Eliciting reliable and relevant VR design tool requirements from designers is challenging because of the lack of experience with and knowledge of VR technologies. This

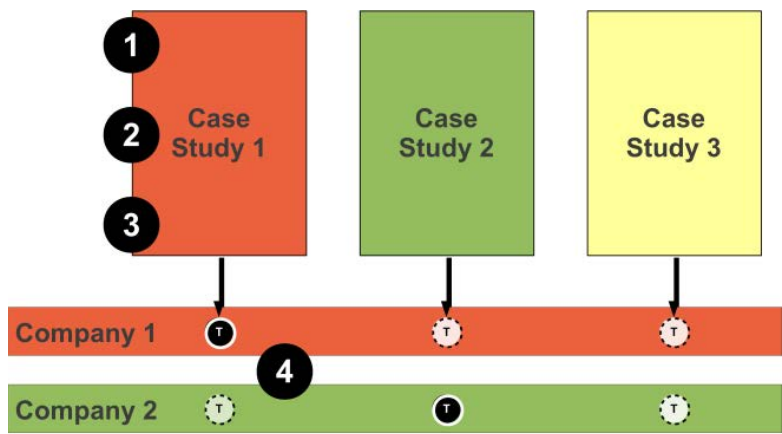

Company $3 \quad \because \div$

VR Solution specifically designed for this company

$\because$ : VR Solution translated to other companies' process

Figure 3: Research approach: three sequential case studies. Each case study features (1) Identification of Application, (2) Providing Design Tools and (3) Deployment and Evaluation. A cross-company validation and generalisation takes place after each case study (4)

is a known issue in VR development [8. To cope with this, our approach is very much application-oriented. Here application refers to how a particular VR technology is used in the design process. For instance, augmented reality can be applied as a virtual prototyping method. Identifying such an advantageous application of VR within the design process provides product designers with a familiar and relevant frame of reference from which they can derive tool requirements. The user-centred and applicationoriented approach is implemented in three sequential industrial case studies (see Fig. 3). Each case study comprises the following steps.

- Identify Applications - Participants are introduced to a wide range of VR technologies and their potential application in a design process. Guided by the researcher (e.g. through workshops) the participants match technologies to opportunities in their company's product development process.

- Provide Design Tool - With the application in mind, the researcher provides the participants with VR design tools (these tools are used as probes) that enable designers to create and use the envisioned application.

- Deploy \& Evaluate Design Tool - Participants use the VR design tool in a test case, and evaluate the design tool as well as the application (e.g. does the application indeed offer the anticipated added value, and does the tool allow us to easily create the application?).

The above steps are carried out within the scope of each specific case study. The company-specific results are validated and generalized through a cross-company evaluation, to find out how well the application and tool can be translated to contexts in other companies. For instance, a tool may perfectly fit the tool chain of company A, but require modifications to also work for company B. 
Doing these cross-company evaluations for all three case studies will lead to insights in the applicability of requirements across industries. The remainder of this section presents the detailed approach of the first case study.

\section{Case Study}

The first case study involved designers from Company B, a multinational manufacturer of printers and copiers. The participating designers were all part of the R\&D department of this company, but ranged from visual designers to usability experts and interaction designers. The following subsections highlight two parts of the case study where involvement of designers was most prominent.

\section{Identify Applications}

The application-oriented approach requires a proper description of an advantageous VR application for the company. Finding such applications requires insights in the company's design process as well as knowledge of the state of the art in VR technologies. The researcher and designers exchanged their respective expertise in a group workshop.

The workshop used visual storyboards to facilitate communication between the researcher and the designers. Twelve designers were asked to visualise typical design scenarios in storyboards. Tangible cards (or frames) were provided to ease the creation of the storyboards. These frames visually depicted design activities, such as 'interviewing an end user' or 'working on a CAD model'. In addition to the regular activities, designers were provided with 'technology frames' representing various VR technologies. The frames included a visual and a brief textual explanation of the technology (leaving room for interpretation by the designers). Designers were asked to insert these technologies into the regular design scenarios wherever the seemed fit. After reviewing the resulting twelve individual storyboards, the designers formed four groups. Each group was asked to create a more detailed final storyboard, in which details about the people, tasks, goals and tools in each frame were added.

The four resulting group storyboards visualized situations in the design process where VR applications were considered useful. The contribution of the storyboard workshop did not lie in the novelty of these applications, but rather in giving VR technologies a familiar context, which made it easier for designers to identify and discuss relevant requirements for the application.

After a review and voting round there was consensus about which application to focus on. The designers envisioned a virtual environment in which a virtual product concept could be tested by end users. A physical environment for this purpose was already available within the company, but it did not resemble the typical use context of end users (e.g. print shops). A realistic virtual use context was expected to trigger more reliable behaviour and feedback from a test user during a concept evaluation.

\section{Application Refinement}

To further refine the characteristics of this application, a one day hands-on session was organized. The session presented four different implementations (prepared by the researcher) of the application. All four implementations consisted of a virtual office environment in which designers could walk around and interact with objects. The four implementations used two different levels of realism (high and low realism environments) and two different levels of virtuality (a fully virtual environment and an augmented reality environment). Designers were asked to carry out a concept evaluation in each of the implementations, allowing them to experience the effects of different levels of realism and virtuality. This hands-on experience helped with narrowing down the focus of the application. It was found that the application primarily facilitates analysis of high-level task flows (e.g. end users moving from one place in the office to another to operate machines) rather than lower level interactions, such as user-machine interactions.

\section{Towards Tool Requirements}

With the application clearly defined, the remainder of the case study focused on determining which tools to use for creating the application, and how to let these tools fit the tool chains and skills available within the company. Designers were involved in this part of the case study in two ways. Firstly, they compared the tool chains that were used for the four hands-on implementations to the tool chains currently used within the company. This identified opportunities to re-use resources already available within the company (e.g. CAD tools or model repositories) and pinpoints bottlenecks in the tool chain (e.g. missing links in the current tool chains). Secondly, the designers were involved in resolving these bottlenecks by evaluating the new links in the tool chain. These evaluations provided insights in the required functionality and usability of VR design tools. As said, the results of these case-specific activities are validated and generalized by a cross-company evaluation carried out after completion of the case study.

PART III: SUPPORTING THE DESIGN PROCESS THROUGH TOOLS AND GUIDELINES: FIRST RESULTS

In this section we present preliminary results and insights about how the early stages of the design process may be supported by tools and guidelines. In part IIIa we present opinions of designers about how the tools under development may support the design process. In part IIIb we present a study how different types of representations that can be generated by the tools may be applied in the design process to elicit feedback from potential end users.

\section{IIla: Insights from Tool Development}

As was mentioned in the introduction to Part II, the working hypothesis is that the two tools under 
development are suitable for different stages in the design process: Sketchify was assumed to be more suitable for the very early stages of the design process, where designers are generating and exploring first ideas, while the VR tool appeared more suitable for the following stage, where the concepts are explored in more detail and where the fit-tocontext is explored.

\section{Insights concerning Low-fi Prototyping Tools}

The workshop that was organized to elicit opinions about the low-fi prototyping tool provided useful feedback and insights. Participants confirmed the general usefulness of Sketchify in the early stages of the design process. They liked the fact that the tool allowed the user to create sketchy-looking drawings and paints, since this is what they normally do in the early stages of the design process when the concepts are not entirely defined.

In addition, participants were also able to describe more detailed future use cases. The ability of the tool to quickly and inexpensively materialize ideas into artefacts was considered useful for both internal (within the design team) and external communication (with end users). A feature that was considered very useful was the tool's ability to support the exploration of complex ideas that require connectivity of software services, or hardware components like Arduino boards, controlling actuators or sensing data. "It would be very interesting if it were indeed really simple to connect any other kind of custom hardware, if it was prepared for that, or if we could just write an adapter once and use it from there on". One usability engineer was very pleased with an example that combines a graphical user interface on the computer screen with a physical mock-up of a product that included LED lights. Currently, when doing early concept evaluations designers do not have a way to transcend the screen and bring more aspects of the experience to the world outside the computer. "It is interesting that your examples simulate not only the user interface, but also part of the outside world"; "I would find such an example very useful and believe that it can bring additional experience to my usability tests". Another example interconnected various computers and beamers to recreate an experience of a futuristic room with dynamic lighting properties. This example attracted much interest by the participants; "We could definitely use something like this, I can't think of another way to do this. Regular sketching would not allow us to see how projections dynamically change and to see immediately how it works, and even be able to change things in the meanwhile".

Important requirements for such use cases are related to the flexibility of the tool, and for it to be simple enough to quickly express and let people understand ideas during discussions and brainstorms: "A simple sketching tool with interaction, easy and fast enough to use during a discussion would be of great value". "PowerPoint is too limited and Sketchflow takes too much time". "The beauty of the software is its simplicity. If you can't use it after 20 minutes of learning I don't reckon using it in a brainstorm session". "An alternative would be to have someone select the best ideas and prepare them outside the discussion, after the brainstorm, and then at the end of the day bring the group together again to continue". However, for the envisioned use cases the threshold of the current tool, for instance with respect to programming, should be further reduced, as was evident from the following remark: "You don't have to program, but it requires a programming mindset", which may be too much of a threshold for some designers.

\section{Insights concerning High-Fidelity Prototyping Tools}

In the hands-on workshop session explained in section 2 designers were able to experience a VR application they had identified during a prior group workshop. The application involved a virtual use context (in this case a print shop environment) in which virtual product concepts could be 'experienced'. During the workshop a simple test case concerning the re-design of a physical printer component was introduced. Four concept alternatives for this component were evaluated in a virtual environment. The session featured four implementations of the virtual environment. Each implementation represented a different combination of the following application parameters.

- Level of realism (low or high) - The level of realism comprises of visual realism, auditory realism, the absence or presence of animations and the behaviour of virtual objects.

- Level of virtuality (fully virtual or augmented reality) - The fully virtual environment consists of a 3D virtual environment of which the context, the objects and the field of view are projected on a large screen (similar to a $3 \mathrm{D}$ first person game). The augmented reality environment consists of a hand-held computer that shows a mixed view of real-time camera images and augmented virtual information (e.g. objects).

Together, the implementations provided four different ways of experiencing the virtual environments. For instance, the augmented reality environment allowed designers to physically move around a virtual office, while the fully virtual environment was controlled through traditional input devices. After going through the concept evaluations in all four environments, the four participating designers were able to reflect on the application parameters.

Firstly, there was a clear preference for the fully virtual environment over the augmented reality environment. The AR environment failed to keep the participants 'immersed' in the context because the augmented world was only visible when looking at the hand-held computer: "Objects only pop up when I'm in range, and just on the small 
screen". With the fully virtual environment, the designers were constantly engaged in the context, despite the lack of mobility.

Secondly, it was found that the virtual environment does not necessarily need to be a 1:1 copy of a real-life context. For instance, the low-realism environment was considered just as 'familiar' as the high-realism environment; a chair should be recognisable as a chair, but it does not need to have the same colour, texture and shape as a chair in the real-life use context (as was the case in the high-realism environment). There is however a lower-bound to the level of realism: "You need a visual clue to know where to click on the printer". Detailed textures and shadings help with estimating object dimensions, and simple shadows add to the sense of objects 'standing' on a floor. When using the low-realism environment, participants for instance failed to notice an empty box lying on the floor causing them to 'tip' over the box. In the high-realism environment, the box had a proper shadow and was more easily detected by participants.

Thirdly, carrying out the evaluations made clear that the added value of this type of VR application is not in detailed concept explorations but rather in workflow analysis: "This [fully virtual] setup is really good in showing the workflow, and the effects of specific tasks on the context, such as the waiting queue". The virtual environment allows end users to experience how the future product interacts with other (future) products, and how the context affects the use of the new product. In our hands-on session we simulated a printer running out of paper, requiring the virtual user to get new paper and refill the printer. Such higher level tasks are not taken into account in the company's existing evaluation methods.

\section{Conclusion}

In general, the results so far support the view that the tool for creating low-fi prototypes and the envisaged tool for creating hi-fi (VR) prototypes can indeed support concept exploration and development in different stages of the concept development process. It must be added, though, that no direct comparison was made between the tools. In particular, because the state of development of the tools is different, it is not possible at the moment to have designers in companies use both tools in their everyday design processes and provide feedback about how and when they would apply the tools. Therefore, in future research, we will have to determine an alternative approach to see how the tools may complement each other, and find out how they can be applied as part of the practitioners' tool chain.

\section{IIIB: Eliciting different types of feedback through} different types of concept representations

A first indication of how different representations might enable designers to elicit certain types of feedback in the designer-user dialog was obtained by a study described in this subsection.
In order to gather early, relevant and reliable user feedback, the designer is in need of media to enable him or her to communicate the concept proposal with the end user, giving the end user a true sense of what the product is and what its benefits and capabilities are 26. In the later phases of the design process, establishing such a dialogue is easier since the designer has a clearer idea about what the product is going to be, and how it can be represented more precisely, such as through working prototypes. However, if the feedback is negative at this stage, it means that a considerable amount of the work carried out to create the prototype was useless. Therefore, it is equally important to enable an effective designer-user dialogue in the earlier phases of the design process when the decisions are not yet concrete. However it is harder to establish that dialogue, as the idea of the designer is not yet fully articulated.

In order to use the tools for creating concept representations effectively, designers should have an idea about the relation between qualities of the concept representations and the type of feedback they elicit. Motivated by these concerns, we conducted a study, in collaboration with one of the industrial partners, to investigate the effect of different types of concept representations on the type of user feedback. A concept of a really new product which proposes new capabilities and new use patterns was chosen as a case for the study.

Visual quality and animation were manipulated to form design representations of different quality. The same concept was visualized both in the form of a storyboard and in the form of an animation, where the transitions between the key frames were animated through fade-in and fade-out. Both for the animation and the storyboard a sketchy and a visually more detailed and refined representation were prepared. It was believed that the look of the representations and the visualization of transitions between key frames will have an influence on the provided feedback. A $2 \times 2$, between-subjects design was applied, with 12 participants per group. Both quantitative and qualitative measures were utilized in the study.

The results of the quantitative analysis did not indicate any particular contribution of visual quality and animation on the understanding of the concept by the participants [31], their judgments on the appeal and hedonic qualities of the concept [12] and whether they could imagine the concept and imagine themselves as a user of the concept [11][31]. However, the quantitative analysis revealed that visual quality had a significant effect on the confidence of the participants that the concept can be realized in real life $\left(\mathrm{F}(1,40)=4,28, \mathrm{p}=.04\right.$, partial $\left.\mathrm{y}^{2}=.097\right)$. Accordingly, participants who saw the concept through realistic representations were significantly more confident that the concept could be realized than the people who saw the concept through sketchy representations. 
The qualitative data were gathered through interviews. Participants were asked about their past experiences, the feedback that they could provide on the concept and also suggestions that they could think of in order to improve the concept. The data were transcribed and analyzed through a verbal analysis method 77 . According to the results of qualitative analysis, animation did not have a significant effect. Visual quality appeared to have an effect on the amount of feedback and also on the number of suggestions. Sketchy representations were found to elicit more elaborate feedback and suggestions and also to trigger more comments relating to concept-related past experiences. On the other hand visually refined representations were good at leading to clear judgments.

This study has given an indication of what kind or reactions different qualities of the design representations evoke. Thus the outcomes of the study might guide designers to prepare concept representations to elicit certain types of feedback. Moreover the study also indicated that well prepared early concept representations can be powerful means to gather deep user insights and elaborated feedbacks and suggestions.

It still remains an open question what kind of feedback is considered useful by designers in the early stage of the design process. Thus a follow-up study is planned with the data of this study, where designers will be confronted with the different types of gathered feedback and will be asked whether and how this feedback will be valuable for the design process.

\section{CONCLUSION}

We have presented work in progress on the development of tools to support the early stages of the design process, especially targeted at the creation and exploration of new concepts. The results so far strengthen the belief that Sketchify and the VR tools under development may be used as part of a tool chain, and might be used both for concept exploration within the design team and cocreation or co-reflection sessions with potential end users. Investigations of the design practice indicated that companies have trouble particularly with the second type of exploration: exploring concepts together with potential end users. It is obvious that just providing tools will not be sufficient to solve this problem. Instead, good tools need to be complemented with guidelines that describe how to use them and paradigmatic examples of how to involve users in the early stages of the design process. Our further research will therefore target both, the further development of the tools, and the development of guidelines for how to use them in the design process. The study on how certain types of concept representation may be used to elicit certain types of feedback is just a first step in this direction.

\section{ACKNOWLEDGEMENTS}

The work reported in this paper was made possible through a grant from the Dutch Government under the IOP-IPCR Programme (Innovation-oriented Research Programme- Integrated Product and Concept Realization). We gratefully acknowledge the contributions of JeanBernard Martens, and Zeljko Obrenovic of Technische Universiteit Eindhoven and Mascha van der Voort of Universiteit Twente, and the people in the companies for diverse contributions in different stages of the research.

\section{REFERENCES}

1. Avrahami, D., Hudson, S. E. (2002). Forming interactivity: A tool for rapid prototyping of physical interactive products. In Proceedings of the 4th Conference on Designing Interactive Systems: Processes, Practices, Methods, and Techniques (DIS'02). ACM,

2. Bailey, B. P., Konstan, J. A., Carlis, J. V. (2001). DEMAIS: Designing multimedia applications with interactive storyboards. In Proceedings of the 9th ACM International Conference on Multimedia (MULTIMEDIA'01). Vol. 9, ACM,

3. Balcisoy, S., Kallmann, M., Fua, P., Thalmann, D. (2000) A framework for rapid evaluation of prototypes with augmented reality. In Proceedings of the ACM symposium on Virtual reality software and technology, Seoul, Korea: ACM, 61-66

4. Bierbaum, A., Just, A., Hartling, P., Meinert, K., Baker, A., Cruz-Neira, C. (2008) VR juggler: a virtual platform for virtual reality application development. In Proceedings of ACM SIGGRAPH ASIA 2008 courses, Singapore: ACM, 1-8

5. Bill Buxton. (2007). Sketching User Experiences: Getting the Design Right and the Right Design. Morgan Kaufmann.

6. Carrol, C., Marsden, P., Soden, P., Naylor, E., New, H. and Dornan, T. (2002). Involving users in the design and usability evaluation of a clinical decision support system. Computer Methods and Programs in Biomedicine. Elsevier.

7. Chi, M.T.H. (1997). Quantifying qualitative analysis of verbal data: A practical guide. The Journal of Learning Sciences, 6(3), 271-315.

8. Cobb, S.V.G., D'Cruz, M.D., Wilson, J.R. (1995) Integrated manufacture: A role for virtual reality? International Journal of Industrial Ergonomics, Vol. 16, No. 4-6, 411-425

9. ISO 13407. (1999). Human-centred design process for interactive systems. International Standard EN/ISO 13407:1999 
10. Goldschmidt, G. (1994). On visual design thinking: the vis kids of architecture, Design Studies, Volume 15, Issue 2.

11.Green, M.C. \& Brock, T.C. (2000). The role of transportation in the persuasiveness of public narratives. Journal of Personality and Social Psychology, 79(5), 701-721.

12.Hassenzahl, M., Platz, A., Burmester, M. \& Lehner, K. (2000). Hedonic and ergonomic quality aspects determine a software's appeal. CHI Letters, 2(1), 201208.

13.Hartman, B., Klemmer, S. R., Bernstein, M., Abdulla, L., Burr, B., Robinson-Mosher, A., Gee, J. (2006). Reflective physical prototyping through integrated design, test, and analysis. In Proceedings of the 19th Annual ACM Symposium on User Interface Software and (UIST'06). ACM,

14. Kara, L. B, Shimada, K. (2007). Sketch-Based 3D shape creation for industrial styling design. IEEE Comput. Graph. Appl. 27, 1, 60-71.

15.Kato, H., Billinghurst, M. (1999) Marker tracking and HMD calibration for a video-based augmented reality conferencing system. In Proceedings of the 2nd IEEE and ACM International Workshop on Augmented Reality, California, USA, 85-94

16.Kuutti, K., Battarbee, K., Säde, S., Mattelmäki, T., Keinonen, T., Teirikko, T., Tornberg, A. (2001) Virtual Prototypes in Usability Testing. In Proceedings of 34th Annual Hawaii International Conference on System Sciences, 5029-5036

17.Landay, J. A. (1996). SILK: Sketching interfaces like krazy. In Conference Companion on Human Factors in

18.Laurel B. (2003). Design Research: Methods and Perspectives. Massachussets: MIT Press.

19.Lin, J., Newman, M. W., Hong, J. I., Landay, J. A. (2000). DENIM: Finding a tighter fit between tools and practice for Web site design. In Proceedings of the ACM SIGCHI Conference on Human Factors in Computing Systems (CHI'00). ACM,

20. Myers, B., Hudson, S. E., Pausch, R. (2000). Past, present, and future of user interface software tools. ACM. Trans. Computer-Human. Interaction

21.Obrenovic, Z. and Martens, J.B. (2011) Sketching interactive systems with sketchify. ACM Trans. Comput.-Hum. Interact. 18, 1, Article 4 (May 2011)

22.Pering, C. (2002). Interaction design prototyping of communicator devices: Towards meeting the hardwaresoftware challenge. Interact.
23.Purcell A.T., Gero J.S. (1998) Drawings and the design process: A review of protocol studies in design and other disciplines and related research in cognitive psychology, Design Studies, Volume 19, Issue 4.

24. Reitmayr, G., Schmalstieg, D. (2001) An open software architecture for virtual reality interaction. In Proceedings of the ACM symposium on Virtual reality software and technology, Baniff, Alberta, Canada: ACM, 47-54

25. Sanders, E.B.N. (1999). Post design and participatory culture. Paper presented at the Useful and Critical: the position of research in design. (Tuusula, Finland, September 9-11).

26. Sleeswijk-Visser, F., Stappers, P.J., van der Lught, R, $\&$ Sanders, E.B.N. (2005). Contextmapping: Experiences from practice. CoDesign: International Journal of CoCreation in Design and Arts, 1(2), 119149.

27. Sleeswijk-Visser, F. (2009). Bringing the everyday life of people into design. PhD Thesis. TU Delft.

28. Stahovich, T.F., Davis, R., Shrobe, H. (1998). Generating multiple new designs from a sketch. Artificial Intelligence 104, 211-264

29. Tideman, M., van der Voort, M., van Houten, F.J.A.M. (2008) A new product design method based on virtual reality, gaming and scenarios. International Journal on Interactive Design and Manufacturing, Vol. 2, No. 4, 195-205

30. Van den Hende, E.A., Schoormans, J.P.L., Morel, K.P.N, Lashina, T., van Loenen, E. \& de Boevere, E.I. (2007). Using early concept narratives to collect valid customer input about breakthrough technologies: The effect of application visualization on transportation. Technological Forecasting and Social Change, 74(9), 1773-1787.

31. Verganti, R. (2009) Design-driven Innovation. Boston: Harvard Business Press.

32. Vredenburg, K., Isensee, S., \& Righi, C. (2002). Usercentered design: An integrated approach. New Jersey: Prentice-Hall.

33. Wakeford, N. (2004). Innovation through peoplecentred design-lessons learned from the USA. DIT global watch mission report.

34. Wright, T.E., Madey, G. (2009) A Survey of Technologies for Building Collaborative Virtual Environments. The International Journal of Virtual Reality, Vol. 8, No. 1, 53-66 